\title{
New quantum diodes with superconducting properties at non-freezing temperatures, and their computing applications
}

\author{
Shinichi Ishiguri, Ph.D. \\ Nihon University \\ 1-2-1 Izumi-Cho, Narashinoshi, Chiba 275-8575 JAPAN \\ TEL: +81-47-474-9143
}

Email: shinichi.ishiguri@gmail.com

\begin{abstract}
Two opposed $\mathrm{p}-\mathrm{n}$ diodes are connected with another junction that causes cancellation of the electric field in the depletion layer of each diode by the field of the other diode. This derived quantum diode is called the A system. Another dual diode, constructed by the same process but with the $\mathrm{p}$ - and $\mathrm{n}$ types positioned as duality, called the B system. When a bias voltage is applied between the A and B systems, Lorentz conservation imparts a momentum (i.e., a wave number) to the carriers in the absence of any internal voltage. Thus, a superconducting bias current density appears without the need for cooling. The reappearances of electron-hole pairs on the junction surfaces are assumed to be described by entire wavefunctions normalized by the band gap. Based on the bias superconducting current, NOT and NAND gates were constructed from the quantum diode systems. Numerical calculations revealed that the constant phases of the entire wavefunctions of the p-and n-types converged. Accordingly, it was clarified that Bose-Einstein condensation and the Meissner effect (described by the London equation) occurred in the quantum diode systems. Moreover, the systems exhibited rectification characteristics and a switching speed of the order of $10^{-14} \mathrm{~s}$. Combining this switching property with the large bias superconducting current (of the order of several V), we developed NOT and NAND gates with direct quantum correlations among many qubits, which are unaffected by random and thermal noises. These gates have memorization and initialization properties and are compatible with existing and accumulating programing algorithms. Moreover, when harvesting a divergent current output from these systems, the bias superconducting current and memorization property preserve the formed quantum correlations.
\end{abstract}

Keywords: quantum diode, quantum gate element, novel superconductivity, bias current, memorization property, quantum correlation 


\section{Introduction}

\subsection{Brief summary of contents}

This paper describes new type of quantum diodes with superconducting properties at non-refrigeration temperatures, and discusses their computing applications. The developed quantum diodes can overcome most of the current problems in quantum computing. This paper is also relevant to condensed matter physics, because the quantum diodes produce a new type of superconductivity at room temperatures.

\subsection{Background part 1 (quantum computing)}

\subsubsection{Moore’s law}

Moore's law is a well-known rule in the computing world. Following this law, companies and researchers have been increasingly downsizing the sizes of transistors and other electronic elements, enabling the package of many elements into small-sized devices, and improvements in specifications. Thus far, downsizing has been relatively easy because the carriers in elements are governed by macroscopic physics. However, further increase in the number of elements and size reduction will require consideration of the microscopic physical equations and laws, which apply to atom-sized structures. Within this quantum realm, computing elements cannot be designed by conventional and existing methods.

\subsubsection{Lowered energy consumption}

Another problem in producing downscaled computing devices is large-scale energy consumption and losses. According to a current estimate, approximately $10 \%$ of human activities are invested in computers or IT devices [1]. Many computing famous companies such as Google and IBM are attempting to reduce their energy consumptions to mitigate $\mathrm{CO}_{2}$ emissions, which are largely responsible for the greenhouse effect. However, as demands for IT and computing devices continue to expand, these companies might need to completely replace their existing computing devices.

\subsubsection{Merits of quantum computing}

The above problems might be solved by a quantum computer that simultaneously solves several problems through quantum correlations. Quantum computing can dramatically reduce the number of 
elements in a device. Current quantum computers are divisible into gate and annealing types. However, many problems must be overcome before quantum computers enter mainstream use. Let us consider the problems facing each category of quantum computers.

\subsubsection{Gate methods}

We first consider problems in gate-type quantum computing methods.

Unlike conventional computing, quantum computing relies on quantum correlations; that is, the interactions among wavefunctions. Without these correlations, quantum computing becomes meaningless and high specifications cannot be achieved. To avoid the difficulty of making many direct correlations, researchers have attempted to make indirect correlations using the existing gate elements, photon, spins, and other known features [2]. However, indirect correlations inevitably increase the overhead and prevent compact downsizing. To exploit the merits of quantum computing, many direct quantum correlations are needed.

The second problem is noise. Because quantum gates originate from microscopic physical parameters such as spins, they are generally very sensitive to thermal noises. The performance of a quantum computer is often defined by its error rate. The error rate of quantum correlations is still high (typically of order $10^{-3}$ ) [3-6]. If many quantum gates are constructed within a small space, superposition drives the error rate toward 1.0.

The third problem stems from the clone prohibition theorem. Data in existing computers are commonly copied to guarantee the validities of programming and clock synchronization. Data copies are equally important in quantum computing, but are excluded by the clone prohibition theorem. Quantum algorithms that resolve this problem are only provisional at this stage.

The final problem is loss of the quantum correlations when harvesting an output. As a wavefunction is related to the uncertainty relation between momentum and position, it is generally considered that when an output is observed, the momentum information is immediately lost, implying that the wavefunction's combination and correlation are collapsed. Quantum algorithms can potentially solve this problem, but are also provisional.

\subsubsection{Annealing methods}

Let us now consider annealing methods, which are rooted in quantum statistical physics such as the Ising model. In annealing methods, noise effects are not concentrated in one element but are averaged over the entire scale. This approach provisionally resolves the noise problem but is limited to specialized cases. The "D-wave machine" model recognizes the technical merit of annealing schemes. D-wave machines have achieved some remarkable progresses [7-8] over existing computers, but 
several reports have highlighted the disadvantages that would be removed even by existing computers. These disadvantages are listed below.

1. Annealing methods are designed to solve specific optimization problems, and are not generic. Some researchers claim that expanding the specifications of annealing methods would impart generic properties. However, very complex procedures and contrivances are needed, which are not suitable for societal spreading.

2. Annealing methods are applied to optimization problems. However, many problems are difficult to recast as optimization problems.

3. Finally, because a D-wave machine employs conventional refrigerated superconductors, it requires cooling apparatus with expensive running costs. Employing this machine many times increases the electric power consumption and $\mathrm{CO}_{2}$ emissions. Therefore, its spread would counteract our current solutions to energy problems.

\subsection{Summary of our proposed device}

The proposed device is simple: prepare two $\mathrm{p}-\mathrm{n}$ diodes, and connect them by a further junction that causes co-cancellation of the electric field in the depletion layers of the original $\mathrm{p}-\mathrm{n}$ junctions. Because the electric fields vanish, the electrons and holes are re-generated in the depletion layers. Moreover, the carriers possess momentum in the absence of voltage in the depletion layers, implying superconductivity. Under a relatively large electric potential, the carriers acquire large momentums through Lorentz conservation. The superconductivity mechanism (i.e., formation of a Cooper pair and the Meissner effect) will be described later. With these superconducting properties, named the bias electric potential or current, the quantum diode operates as a NOT or NAND gate without requiring refrigeration. Importantly, the quantum diodes can be configured as two systems, named the A and B systems. An electric potential applied to the A system is provided by the voltage source connected to the B system.

Besides the electric potential of the bias, we propose taps for inputting and outputting electric potentials smaller than the bias. When a superconductive system can supply voltages to multiple taps, the quantum states of the taps can be correlated through the superconductivity, generating a qubit [910]. Multiple qubits are easily generated by expanding such systems. These directly generated quantum correlations are expected to exhibit quantum supremacy in a compact space, and (as mentioned above) can be configured as NOT and NAND gates.

Because the bias is several volts in magnitude and the input potential and output current density are of the orders $0.1 \mathrm{~V}$ and $10^{3} \mathrm{~A} / \mathrm{m}^{2}$, respectively, random and thermal noises can be ignored, as their magnitudes are much smaller than these quantities.

Meanwhile, the input voltage (i.e., a correlation) in the system is canceled by the reverse output 
voltage induced by the superconducting bias current, annulling the voltage concept. Moreover, this superconductivity is generated without cooling, and incurs minimal running cost. Thus, the energy losses in our quantum diode systems are almost zero. Only a small electric power is consumed at the moment of harvesting the output current density.

As mentioned above, if the current density is harvested from the output tap, the corresponding input tap receives energy from the input voltage source. Note that these input voltage sources between the $\mathrm{A}$ and $\mathrm{B}$ are prepared independently from the voltage source generating bias electric potentials. This mechanism sustains and memorizes the correlations and quantum states. Thus, the quantum correlations are not destroyed by harvesting the output current density from a tap.

Later, we will demonstrate the rectification characteristics and switching speed of the proposed quantum diodes. The switching speed (of order $10^{-14} \mathrm{~s}$ ) is much faster than in conventional diodes, which are hampered by capacitance factors. This ultra-fast switching, combined with the memorizing property imparted by the superconducting bias current, enables very fast memorization and initialization. Accordingly, the system can accommodate conventional and accumulated programming methods at much faster calculation speeds.

\subsection{Background part 2 (superconductivity)}

\subsubsection{Superconductivity}

This paper proposes quantum diode systems with a new superconductivity mechanism that operates at non-freezing temperatures. We now review the background and history of superconductivity. Typical superconductors are characterized by zero electrical resistance and the Meissner effect. The zero-resistance property greatly reduces the energy consumption, and is now being exploited in superconducting energy storage and electric power transmitting systems [11]. However, superconductivity appears only under strongly cooled conditions and incurs a high manufacturing costs. These disadvantages prevent the wider dissemination of superconductors in practice.

\subsubsection{Brief history of superconductors}

Superconductivity was discovered approximately one century ago, when an $\mathrm{Hg}$ superconductor was refrigerated in liquid $\mathrm{He}(4.2 \mathrm{~K})$. This finding triggered a search for further metal-based superconductors. Eventually, the critical temperature of superconductivity was raised to around $20 \mathrm{~K}$. The Bardeen-Cooper-Schrieffer theory [12], which has been supported by some researches, upperlimits the critical temperature of superconductivity to $30 \mathrm{~K}$. However, ceramic cuprates with critical temperatures higher than liquid- $\mathrm{N}_{2}$ temperature $(77 \mathrm{~K})$ were discovered in 1986 [13]. Since that time, 
novel compounds with higher critical temperatures have been actively sought, culminating in Fe-based superconductors ( $50 \mathrm{~K}$ ) [14], $\mathrm{MgB}_{2}$ metal superconductors (39 K) [15], and H-based high-pressure superconductors [16] (> $250 \mathrm{~K}$ but requiring pressures above $250 \mathrm{GPa}$ ). However, compounds with critical temperatures around room temperature $(300 \mathrm{~K})$ at atmospheric pressures have not been found. The discovery of such room-temperature superconductors is expected to revolutionize the electrical and energy industries.

\subsection{Summary of superconductivity in our proposed device}

As mentioned above, this paper proposes room-temperature superconductivity by a circuit configuration approach rather than seeking new compounds. The entire wavefunction is considered as a superposition of electrons and holes, respectively. In numerical calculations, the convergence of constant phases in the entire wavefunction clarify Bose-Einstein condensation and the Meissner effect (via the London equation). These calculations theoretically verify the appearance of superconductivity.

The search for new superconducting compounds, which is currently undertaken by almost all researchers, is unlikely to reveal superconductors with critical temperatures above room temperature. However, our circuit approach promises an easy solution. Although our literature search revealed several new types of superconductivity at room temperatures and pressures [17-20], we believe that the circuit approach is a breakthrough in the search for superconductivity.

\section{Principle}

As shown in Fig. 1, we connect two p-n diodes in reverse order. In this configuration, the electricfield directions in the depletion layers of the upper and lower diodes oppose each other, and thus cancel at the junction (labeled O-O' in Fig. 1). As the electric field is cancelled, electrons and holes can be re-generated in the depletion layer. Moreover, under the momentum conservation law, these

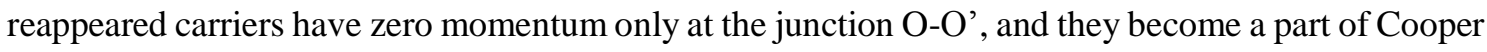
pair as an exciton pair. However, as discussed later, many-body interactions of realms' carriers create a constant phase in the macroscopic wavefunction, implying Bose-Einstein condensation and a net superconductivity, i.e. the Meissner effect. 

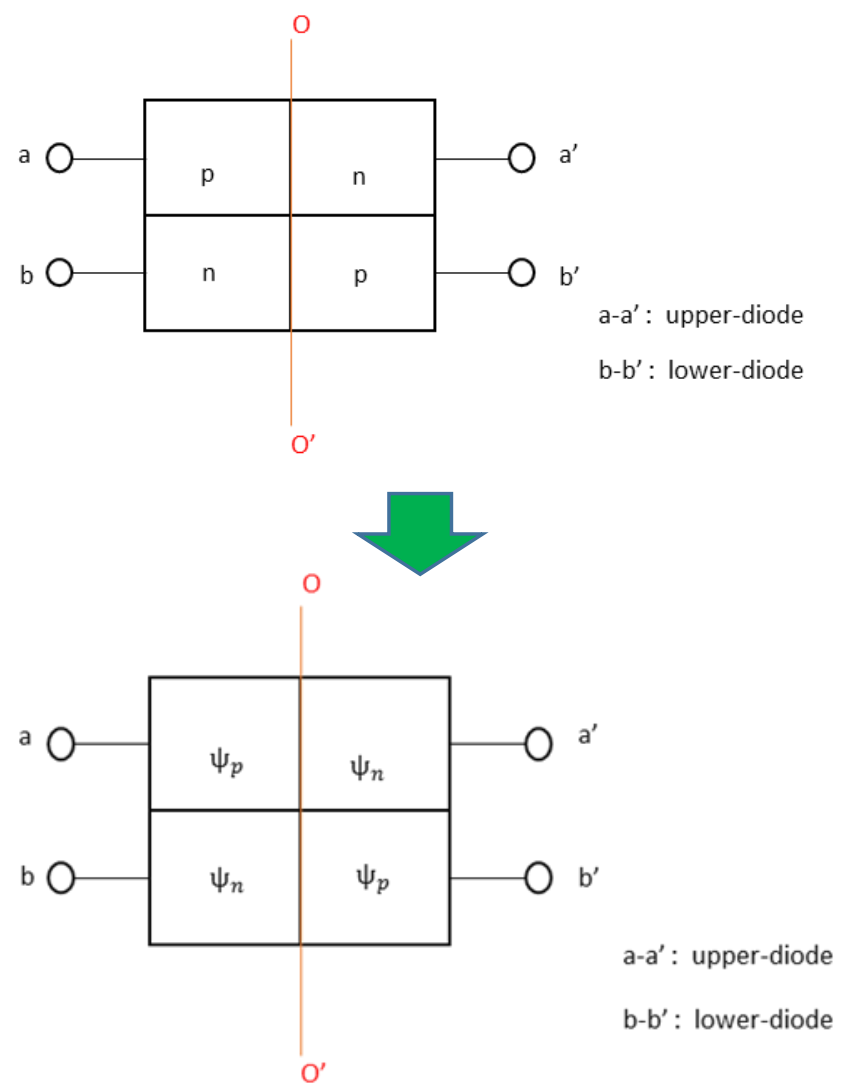

Fig. 1. In this configuration of normal p-n diodes, the electric fields in the depletion layers of both diodes cancel at the junction. Consequently, electron-hole pairs reappear at the junction surface. In the bottom panel, the electrons and holes at the junction are replaced with their macroscopic functions $\psi_{\mathrm{n}}$ and $\psi_{\mathrm{p}}$, respectively.

\section{Theory}

\subsection{Motion equation and electrostatic potential $V_{0}$ at the junction}

Let us consider the conservation of momentum of each quantity, which will result in zero momentum as mentioned. The electric fields of the holes in the upper and lower $\mathrm{p}-\mathrm{n}$ diodes at the proposed junction are given by

$$
\begin{aligned}
& +q(+E)=\frac{d\left|k_{h}\right|}{d t}, \quad \text { (upper diode) } \\
& +q(-E)=\frac{d\left|k_{h}\right|}{d t}, \quad \text { (lower diode) }
\end{aligned}
$$

where $q$ and $k_{h}$ denote the carrier charge and wavenumber of a hole, respectively. 
From these equations, the electrostatic potentials $V_{0}$ at the proposed junction are given by

$$
\begin{aligned}
-q V_{0}=\int \frac{\partial\left|k_{h}\right|}{\partial t} d x, & \text { (upper diode), } \\
+q V_{0}=\int \frac{\partial\left|k_{h}\right|}{\partial t} d x, & \text { (lower diode). }
\end{aligned}
$$

The electrostatic potentials of the electrons at the proposed junction are similarly given by

$$
\begin{gathered}
+q V_{0}=\int \frac{\partial\left|k_{e}\right|}{\partial t} d x, \quad \text { (upper diode), } \\
-q V_{0}=\int \frac{\partial\left|k_{e}\right|}{\partial t} d x, \quad \text { (lower diode) }
\end{gathered}
$$

where $k_{e}$ is the wave number of an electron.

In the upper diode, Eqs. (2-1) and (3-1) are superposed as follows:

$$
q\left(V_{0}-V_{0}\right)=\frac{d}{d t} \int\left(\left|k_{h}\right|+\left|k_{e}\right|\right) d x
$$

In the lower diode, Eqs. (2-2) and (3-2) are similarly superposed as

$$
q\left(V_{0}-V_{0}\right)=\frac{d}{d t} \int\left(\left|k_{h}\right|+\left|k_{e}\right|\right) d x
$$

From these equations, we get

$$
0=d \int\left(\left|k_{h}\right|+\left|k_{e}\right|\right) d x,
$$

Assuming that the first and second terms in the kernel of this expression are both positive, we have

$$
\left|k_{e}\right|+\left|k_{h}\right|=0
$$

and hence

$$
k_{e}=k_{h}=0 .
$$

Therefore a reappeared hole and electron have zero phase at the junction surface O-O', considering that a phase at the steady state is generally expressed as the product of both a wave number and position variable by introducing the Schrodinger eigenvalue equation and its plane wave as a solution of it. That is,

$$
\begin{aligned}
& \psi_{e}=\left|\psi_{e}\right| \exp (i \theta) \approx\left|\psi_{e}\right|, \\
& \psi_{h}=\left|\psi_{h}\right| \exp (i \theta) \approx\left|\psi_{h}\right|,
\end{aligned}
$$

and

$$
\int\left(\left|\psi_{h}\right|^{2}+\left|\psi_{e}\right|^{2}\right) d v=1
$$

Note that this volume integral should be very small because the wave numbers $k_{e}$ and $k_{h}$ are almost zero. 


\subsection{Normalization of the entire wavefunctions}

The total probability density function $|\psi|$ of both carriers is normalized as follows:

$$
\int\left(\left|\psi_{B, h}\right|^{2}+\left|\psi_{B, e}\right|^{2}\right) d v=1,
$$

where $\psi_{B, h}$ and $\psi_{B, e}$ are the total and macroscopic wavefunctions of the holes and electrons, respectively. The next section will describe the details.

The band gap $E_{G}$ in the depletion layer is given by

$$
\hbar \omega_{G}=E_{G},
$$

where $\hbar$ is the reduced Planck constant and $\omega_{G}$ is the corresponding angular frequency. The corresponding wavelength is

$$
\lambda_{G}=\frac{\hbar c}{E_{G}},
$$

where $c$ is the speed of light. The above normalization then becomes

$$
\begin{aligned}
& \int 2|\psi|^{2} d v=2|\psi|^{2} \lambda_{G}^{3}=1, \\
& |\psi|^{2}=\frac{1}{2} \times\left(\frac{E_{G}}{\hbar c}\right)^{3},
\end{aligned}
$$

where

$$
|\psi|^{2} \equiv\left|\psi_{B, h}\right|^{2}=\left|\psi_{B, e}\right|^{2}
$$

For example, when the band gap $E_{G}=1.5 \mathrm{eV}$, we have

$$
|\psi|^{2}=2.2 \times 10^{20} \text {. }
$$

\subsection{Converged constant phases of the macroscopic wavefunctions}

Next let us consider whether the phases of the entire wavefunctions of the electrons and holes are constant and convergent.

First, assume that the probability density function of a hole at the junction O-O' that has no phase interacts with that of a normal electron in the vicinity of the junction.

$\varphi_{B, e}=\left|\psi_{h}\right|+\left|\psi_{e}\right|[\exp (i \theta)]$,

Similarly the probability density function of an electron at the junction O-O' that has also no phase interacts with that of a normal hole in the vicinity of the junction.

$\varphi_{B, h}=\left|\psi_{e}\right|+\left|\psi_{h}\right|[\exp (-i \theta)]$,

where the second term is a solution of the Schrodinger equation, and $i$ denotes an imaginary number. This assumed wavefunction implies an interaction of the probability density functions at the junction, which was discussed in the previous section, and a neighboring carrier, and that this carrier's general 
wavefunction can be approximated as a plane wave. The second terms in Eq. (18-1) and (18-2) have phase

$$
\theta=k_{R} \xi
$$

where $\xi$ and $k_{R}$ denote the constant coherence of a Cooper pair and relative wave number, respectively. Later a discussion will be provided, which combines this coherence and the combination energy among a pair. Thus Eq. (18-1) and (18-2) imply wave functions of a Cooper pair having a relative wave number. Note that, because this phase is variable, a relative wave number $k_{R}$ must be variable. Thus,

$k_{R} \equiv \frac{2 \pi n}{\xi}$,

where $\mathrm{n}$ denote variable integer.

Due to the existence of this relative wave number, the physical pictures to form a Cooper pair are described as:

1. Rotational moving electron around hole having zero momentum with combination by attractive Coulomb force expressed by Eq. (18-1)

2. Rotational moving hole around electron having zero momentum with combination by attractive Coulomb force expressed by Eq. (18-2).

Thus importantly a Cooper pair in the present paper is like an exciton.

Because the wavefunctions (18-1) and (18-2) respectively describe a boson, the entire wavefunction of eletrons is expressed as

$$
\psi_{B, e}=\Pi^{j} \varphi_{B, e}=\Pi^{j}\left[\left|\psi_{h}\right|+\left|\psi_{e}\right| \exp \left(i \theta_{j}\right)\right],
$$

Similarly, the entire wavefunction of holes is

$$
\psi_{B, h}=\Pi^{j} \varphi_{B, h}=\Pi^{j}\left[\left|\psi_{e}\right|+\left|\psi_{h}\right| \exp \left(-i \theta_{j}\right)\right],
$$

Note that in the calculations, the plane waves in the second terms of Eqs. (19) and (20) are replaced by cosine functions.

Figures 2 and 3 are schematics of the entire wavefunctions given by Eqs. (19) and (20), respectively. Accurate calculations will be presented in the Results section. As indicated in the normalization equation, the absolute value of the wavefunction must be nonzero and finite. In the Results section, we will show that the maximum value of Eq. (19) and (20) is the square root of Eq. (15) at a particular constant phase $\pm \theta_{0}$. This implies that the phase convergence of the electron and hole wavefunctions produce Bose-Einstein condensation and the Meissner effect (which signify superconductivity). Details will be presented in the Discussion section. 


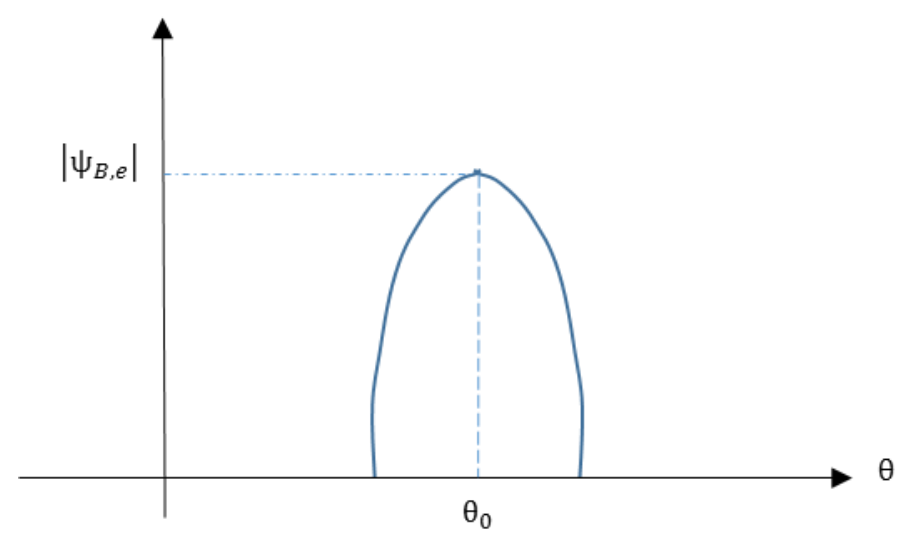

Fig. 2 Phase convergence of the entire wavefunction of electrons.

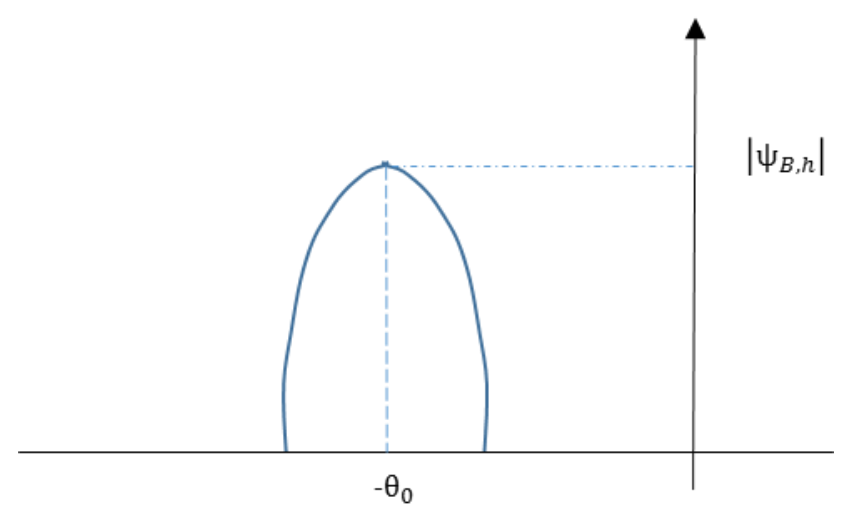

Fig. 3 Phase convergence of the entire wavefunction of holes, which opposes that of the electrons.

\subsection{Bias current density}

\subsubsection{Steady state}

\section{Forward bias case}

Let us consider the bias current density under an applied electrostatic potential $\varphi_{\mathrm{B}}$. By Lorentz conservation (see Fig. 4), we have

$$
(\hbar k)^{2}-\left(\frac{\varepsilon}{c}\right)^{2} \equiv 0,
$$

where the energy $\varepsilon$ of a carrier is given by

$$
\varepsilon=+e \varphi_{B} .
$$

Here, $e$ is the charge of the carrier. 
The above equation implies the forward bias. Substituting Eq. (22) into Eq. (21) the wave number $k$ is derived as

$$
k=+\frac{e \varphi_{B}}{\hbar c} .
$$

Considering the probability density flow, the current density $j_{t 0}$ at steady state is

$$
j_{t 0}=e|\psi|^{2} \frac{\hbar k}{m}=+e^{2}|\psi|^{2} \frac{\varphi_{B}}{m c}
$$

where $m$ denotes the mass of an electron and as mentioned in Eq (15),

$$
|\psi|^{2}=\frac{1}{2} \times\left(\frac{E_{G}}{\hbar c}\right)^{3} .
$$

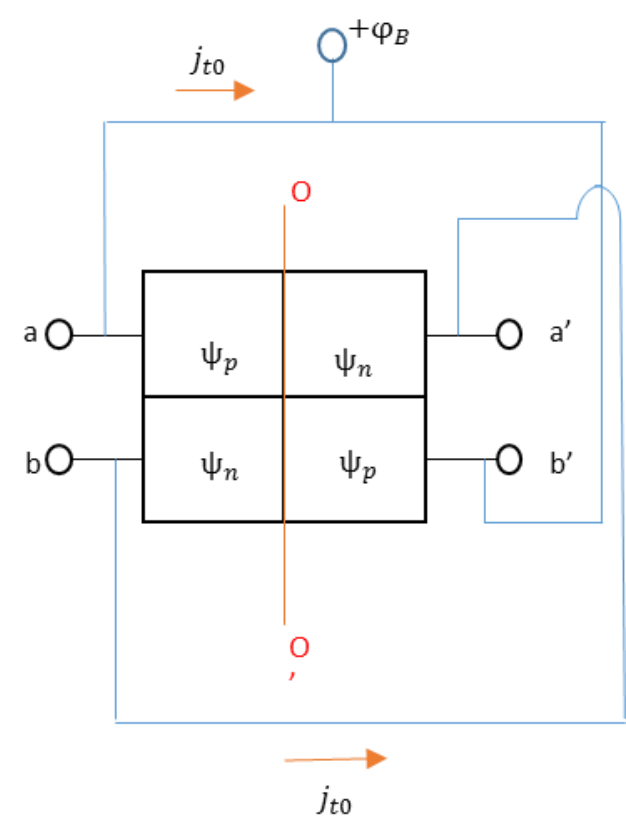

Fig. 4 Application of a bias electrostatic potential $+\varphi_{B}$. Lorentz conservation guarantees a superconducting current density $j_{t 0}$. Note that the electric potential $+\varphi_{B}$ is not a voltage (see Fig. 5). 


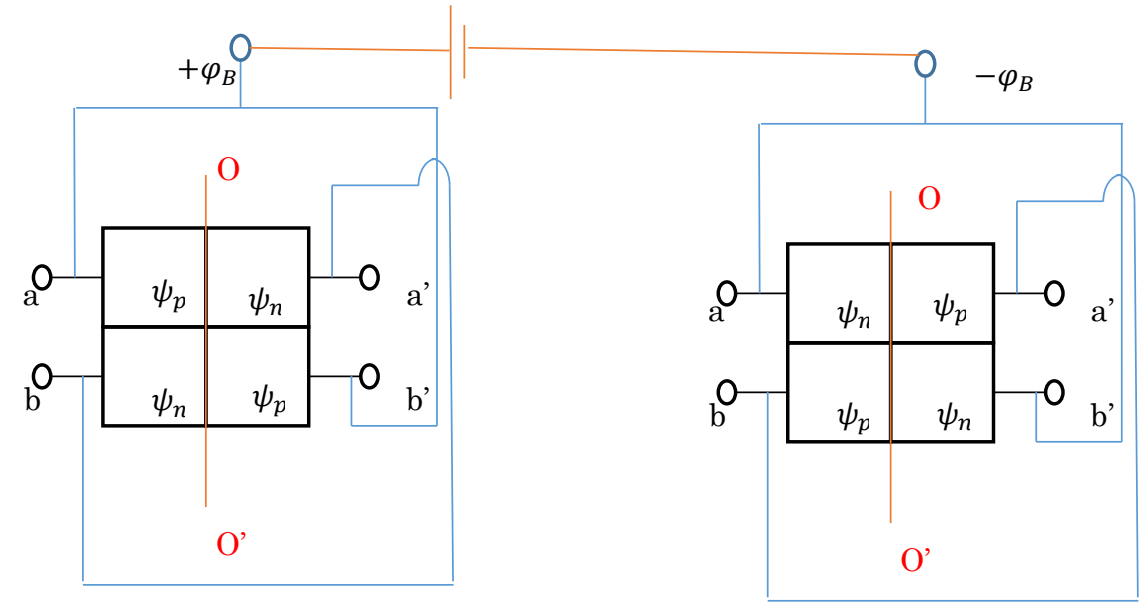

A system

B system

Fig. 5 Schematic of dual systems. Note the different positions of the p- and n-type semiconductors in the two systems. These dual systems act as quantum diodes that apply electric potentials to each system.

Note that the above $+\varphi_{B}$ is an electrostatic potential, not a voltage (see Fig. 5). The A system in Fig. 5 is identical to Fig. 4, but the B system, which opposes rather than corresponds to the p- and n-type semiconductors, remains to be configured. Subsequently, voltage sources are applied between the corresponding taps of the A and B systems.

\section{Reverse bias case}

At $t=0$, the forward bias current density $j_{t 0}$ is assumed by given by Eq. (24). Under this condition, the sign of bias-electrostatic potential is reversed:

$$
\varphi_{B} \rightarrow-\varphi_{B}
$$

Consequently, a reverse current density $j_{r}$ is superimposed on the initial $j_{t 0}$.

Thus, the net current density becomes

$$
j_{\text {total }}=j_{t 0}+j_{r}=+e^{2}|\psi|^{2} \frac{\varphi_{B}}{m c}-e^{2}|\psi|^{2} \frac{\varphi_{B}}{m c} \rightarrow 0 .
$$

In summary, an electrostatic potential should be added to provide a superconducting bias current density. Adding a reverse electrostatic potential then reduces the bias current density to zero. 


\subsubsection{Properties in the transient state}

\section{General notation}

The previous subsection discussed the switching property in steady state. This subsection analyzes the switching properties during the transient state. To this end, we modify the Lorentz conservation Eq. (21) by replacing the term " 0 " in the right-hand-side by the time-dependent momentums.

\section{Transient-state forward bias case}

We first consider the momentum change

$$
\Delta p=-F_{0} \Delta t
$$

where $t$ and $F_{0}$ denote the time and a constant force, respectively.

The force is induced by an electric field $E_{\mathrm{B}}$ as

$$
F_{0}=e E_{B}
$$

Moreover, this electric field is related to an electrostatic potential $\varphi_{\mathrm{B}}$.

$$
\frac{d \varphi_{B}}{d x} \approx \frac{\varphi_{B}}{\xi}=-E_{B}
$$

where $\xi$ denotes the coherence of a Cooper pair (for details, see the Results and Discussion sections). Thus we have

$$
F_{0}=-e \frac{\varphi_{B}}{\xi}
$$

and

$$
\Delta p=\mathrm{e} \frac{\varphi_{B}}{\xi} \Delta t
$$

Now, the Lorentz conservation of Eq. (21) is modified as follows:

$$
(\hbar k)^{2}-\left(\frac{\varepsilon}{c}\right)^{2}=\left(e \frac{\varphi_{B}}{\xi} \Delta t\right)^{2}
$$

where

$$
\varepsilon \equiv+\mathrm{e} \varphi_{B}
$$

Thus,

$$
(\hbar k)^{2}=\left(e \frac{\varphi_{B}}{\xi} \Delta t\right)^{2}+\left(\frac{e \varphi_{B}}{c}\right)^{2}=\left(\frac{e \varphi_{B}}{c}\right)^{2}\left[1+\left(\frac{\frac{e \varphi_{B} \Delta t}{\xi \xi}}{\frac{e \varphi_{B}}{c}}\right)^{2}\right] .
$$

Approximately and conclusively, we have

$$
\hbar k \approx\left(\frac{e \varphi_{B}}{c}\right)\left[1+\frac{1}{2}\left(\frac{c \Delta t}{\xi}\right)^{2}\right] .
$$


The transient-state forward bias is subjected to the following initial condition:

$$
\Delta t=0 \rightarrow k=\varphi_{B} \equiv 0 .
$$

Thus, in this case only, we get

$$
\hbar \mathrm{k}=\left(\frac{e \varphi_{B}}{c}\right)\left[\frac{1}{2}\left(\frac{c \Delta t}{\xi}\right)^{2}\right] .
$$

Considering the equation of probability density flow, the forward bias current density in the transient state becomes

$$
j_{t}=e|\psi|^{2} \frac{\hbar k}{m}=+e|\psi|^{2} \frac{e \varphi_{B}}{m c}\left[\frac{1}{2}\left(\frac{c \Delta t}{\xi}\right)^{2}\right] .
$$

\section{Transient-state reverse bias case}

In this case, the total current is obtained by summing the steady bias current density $j_{t 0}$ (which is assumed to already exist) and the current density $j_{r}$ derived from the reversed bias in Eq. (35), in which the sign of the bias is switched:

$$
j_{\text {total }}=j_{t 0}+j_{r}=e^{2}|\psi|^{2} \frac{\varphi_{B}}{m c}+e|\psi|^{2} \frac{e}{m c}\left(-\varphi_{B}\right)\left[1+\frac{1}{2}\left(\frac{c \Delta t}{\xi}\right)^{2}\right]=-e|\psi|^{2} \frac{e \varphi_{B}}{m c}\left[\frac{1}{2}\left(\frac{c \Delta t}{\xi}\right)^{2}\right] .
$$

The derived current densities in the transient state will be presented in the Results section.

\subsection{Gate elements}

\subsubsection{NOT gate}

The above-mentioned quantum diode was outfitted with four taps named $\left(a_{i}, a_{j}\right)$ and $\left(b_{i}, b_{j}\right)$, as shown in Fig. 6. A bias current density $j_{t 0}$ is assumed in advance. Here, the two diode systems in Fig. 5 are dual to each other. The A and B systems in Fig. 6 correspond to those shown in Fig. 5.

Each $\left(b_{i}, b_{j}\right)$ input receives an electric potential when the two taps are connected between the $\mathrm{A}$ and B systems through two voltage sources, provided that these connecting taps correspond to each other. That is, a method to apply input electric potentials to the taps $\left(b_{i}, b_{j}\right)$ employs the same concept for application of bias potential.

An input vector is expressed as

$$
\psi_{\text {in }}=b_{i} \overrightarrow{0}+b_{j} \overrightarrow{1},
$$

where the voltage $v_{\text {in }}$ between $b_{i}$ and $b_{j}$ must be defined. This voltage is described by the symmetric integral

$$
v_{i n}=\int_{-\alpha}^{\alpha} E d x,
$$


where the origin of the coordinates is located at the $\mathrm{p}-\mathrm{n}$ junction (i.e. at the surface O-O').

Note that $b_{i}=b_{j}$ is prohibited, because a voltage $v_{\text {in }}$ cannot be defined in this case.

The bases in the spaces $\overrightarrow{0}$ and $\overrightarrow{1}$ are respectively defined as

$$
\overrightarrow{0} \equiv \psi_{B, e}=|\psi| \exp \left(-j \theta_{0}\right),
$$

and

$$
\overrightarrow{1} \equiv \psi_{B, h}=|\psi| \exp \left(+j \theta_{0}\right),
$$

where the wavefunctions are the previously discussed macroscopic superconducting wavefunctions, and $\theta_{0}$ and $|\psi|$ denote the macroscopic wavefucntion's phase and magnitude, respectively. Recall that

$$
|\psi|^{2}=\frac{1}{2} \times\left(\frac{E_{g}}{\hbar c}\right)^{3} .
$$

Therefore, the input vector becomes

$$
\psi_{\text {in }}=b_{i}|\psi| \exp \left(-j \theta_{0}\right)+b_{j}|\psi| \exp \left(+j \theta_{0}\right) .
$$

In two-dimensional Hilbert space, the components of this vector are

$\left(\begin{array}{l}b_{i}|\psi| \\ b_{j}|\psi|\end{array}\right)$. Generally, the components of a Hilbert space need not be normalized, so $\left(b_{i}, b_{j}\right)$ can take arbitrary values.

For example, if

$$
\left(b_{i}, b_{j}\right) \equiv(B,-B)
$$

we can write

$$
\psi_{\text {in }}=B|\psi| \exp \left(-j \theta_{0}\right)+(-B)|\psi| \exp \left(+j \theta_{0}\right) .
$$

The output vector implies a voltage $v_{\text {out }}$ with the absolute value of $v_{\text {in }}$ in Eq. (41) but of opposite direction (sign) owing to the superconducting bias current $j_{t 0}$. Importantly, if a voltage is applied to a quantum diode in the system, a reverse voltage must appear to maintain the superconducting state:

$$
v_{\text {out }}=-v_{\text {in }} \text {. }
$$

The output vector between the taps $\left(a_{i}, a_{j}\right)$ is then given as

$$
\psi_{\text {out }}=a_{i}|\psi| \exp \left(-j \theta_{0}\right)+a_{j}|\psi| \exp \left(+j \theta_{0}\right) .=-B|\psi| \exp \left(-j \theta_{0}\right)+B|\psi| \exp \left(+j \theta_{0}\right)
$$

The vectors $\psi_{\text {in }}$ and $\psi_{\text {out }}$ are weighted by the probability $|\psi|$. Note that the coefficient $1 / 2$ appears in Eq. (15). The importance of this coefficient is demonstrated in Fig. 7. In this figure, $b_{i}$ corresponds to $a_{i}$ or $a_{j}$ with equal probability (50\%), and $b_{j}$ also corresponds to $a_{i}$ or $a_{j}$ with probability $50 \%$. Note that the following mapping

$$
\psi_{\text {in }}=b_{i}|\psi| \exp \left(-j \theta_{0}\right) \rightarrow a_{i}|\psi| \exp \left(-j \theta_{0}\right)=\psi_{\text {out }}: \text { Not occur }
$$

is prohibited because the voltages $v_{\text {in }}$ and $v_{\text {out }}$ are undefined so the superconducting property and Eq. (46) are not used.

Thus, the plus signs in the vector equations of $\psi_{\text {in }}$ and $\psi_{\text {out }}$ have an essential physical meaning; 
specifically, they imply the existence of quantum correlations.

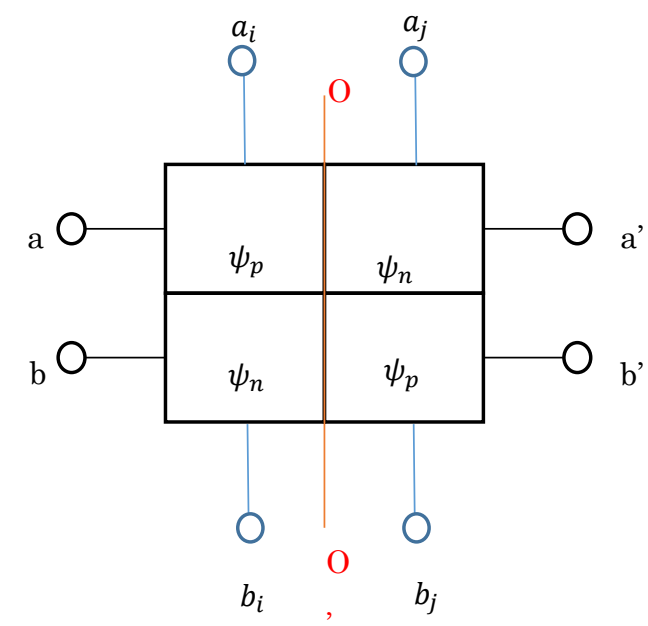

A system

Fig. 6 Schematic of a one-qubit system. In this figure, $\left(b_{i}, b_{j}\right)$ and $\left(a_{i}, a_{j}\right)$ are paired and the dual systems are named the A system and the B system. For inputting a voltage between these systems, electric potentials are applied to each tap in the qubit system. That is, a method to apply input electric potentials is the same concept for the case of the application of bias potential.

To harvest the output electric potentials, each output tap of $\left(a_{i}, a_{j}\right)$ in the A system in Fig. 5 is connected and shorted to its corresponding tap in the B system. Divergent currents then flow and Joule heating losses do not appear. The divergent current flow from the A to the B system implies simultaneous flow of a reverse divergent current from the B to the A system, which passes an input voltage source. Energy conservation holds in this system, but the input $\left(b_{i}, b_{j}\right)$ and output $\left(a_{i}, a_{j}\right)$ can be reversed such that $\left(a_{i}\right.$, $\left.a_{j}\right)$ is input and $\left(b_{i}, b_{j}\right)$ is output; that is, the system is symmetric.

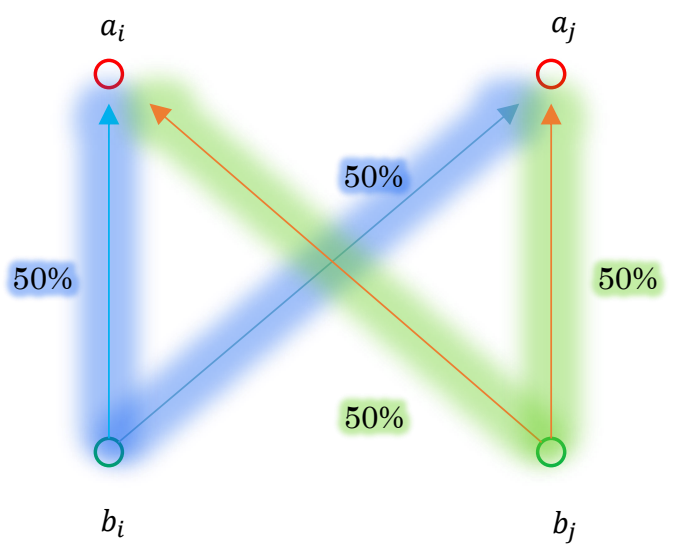


Fig. 7 Schematic of mapping from an input vector's components to an output vector's components. In this figure, $\left(b_{i}, b_{j}\right)$ is a paired component of the input vector in Hilbert space, and $\left(a_{i}, a_{j}\right)$ is a paired component of the output vector. Note that the mapping is not one-to-one.

Let us now consider the two-qubit configuration.

The two-qubit configuration of the A system is shown in Fig. 8. First, the normalization is given as

$$
\int\left(2\left|\psi_{B, e}\right|^{2}+2\left|\psi_{B, h}\right|^{2}\right) d v \equiv 1
$$

Thus we have

$$
|\psi|^{2}=\frac{1}{4} \times\left(\frac{E_{G}}{\hbar c}\right)^{2} .
$$

After obtaining four bases, we describe the quantum correlations among $\left(b_{1}, b_{2}, b_{3}, b_{4}\right)$ in Fig. 8.

The total wavefunction of the electrons is given by

$$
\psi_{B, e} \rightarrow \psi_{B, e}^{\prime}=\Pi^{i}\left(\left|\psi_{B, h}\right|+\left|\psi_{B, e}\right| \exp \left(\mathrm{j} \theta_{i}\right)\right)^{2} .
$$

The total wavefunction of the holes can be given similarly.

This wavefunction is schematized in Fig. 9 (an accurate calculation will be given in the Results section). The corresponding total wavefunction of the holes is depicted in Fig. 10. Note that the wavefunctions in Figs. 9 and 10 are discontinuous at the origin, because one describes the behavior of the electrons and the other describes that of holes. The electrons exist in two quantum states (i.e. two constant phases). This result implies that the n-type with $b_{1}$ and n-type with $b_{3}$ occupy different quantum states. The same phenomena were found in the p-type semiconductors (Fig. 10). When the junction surface O-O' is selected as the origin, the two-qubit quantum state must satisfy Eq. (41):

$$
\psi_{\text {in }}=\left(b_{1} \overrightarrow{00}+b_{4} \overrightarrow{11}\right)+\left(b_{2} \overrightarrow{01}+b_{3} \overrightarrow{10}\right) .
$$

In this equation, the four bases are expressed as

$$
\begin{aligned}
& \overrightarrow{00}=|\psi| \exp \left(-j \theta_{1}\right)=\lim _{\theta_{1 \rightarrow-0}}|\psi| \exp \left(j \theta_{1}\right), \\
& \overrightarrow{11}=|\psi| \exp \left(+j \theta_{1}\right)=\lim _{\theta_{1 \rightarrow+0}}|\psi| \exp \left(j \theta_{1}\right), \\
& \overrightarrow{01}=|\psi| \exp \left(-j \theta_{2}\right), \\
& \overrightarrow{10}=|\psi| \exp \left(+j \theta_{2}\right) .
\end{aligned}
$$

By Eq. (46), the output vector becomes

$$
\psi_{\text {out }}=\left(a_{1} \overrightarrow{00}+a_{4} \overrightarrow{11}\right)+\left(a_{2} \overrightarrow{01}+a_{3} \overrightarrow{10}\right) \rightarrow\left(b_{4} \overrightarrow{00}+b_{1} \overrightarrow{11}\right)+\left(b_{3} \overrightarrow{01}+b_{2} \overrightarrow{10}\right)
$$




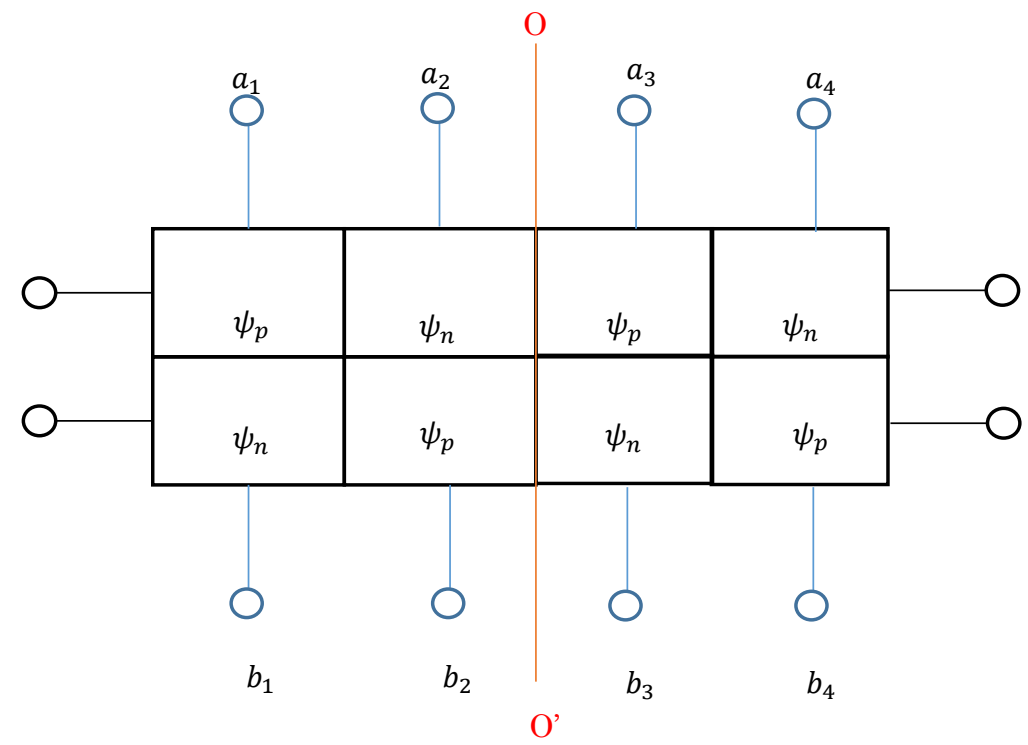

A system

Fig.8

Schematic of the two-qubit configuration. The bias current density $j_{t}$ is assumed to be on. Because the four states are correlated, the magnitudes and constant phases of the entire wavefunction can change (unlike the one-qubit case).

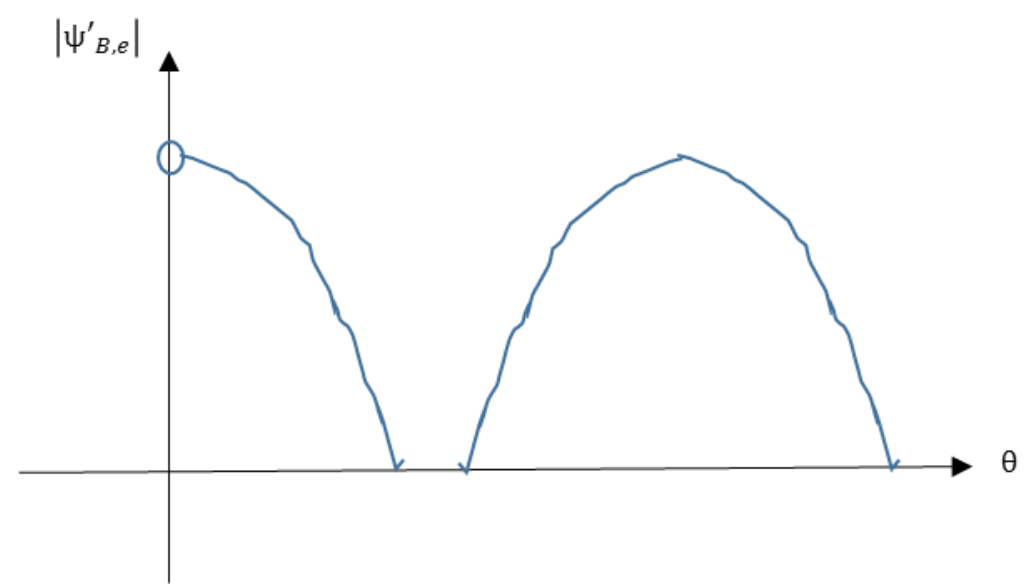

Fig. 9 Schematic of the entire wavefunction of the electrons in the 2-qubit case. Two constant phases appear. 


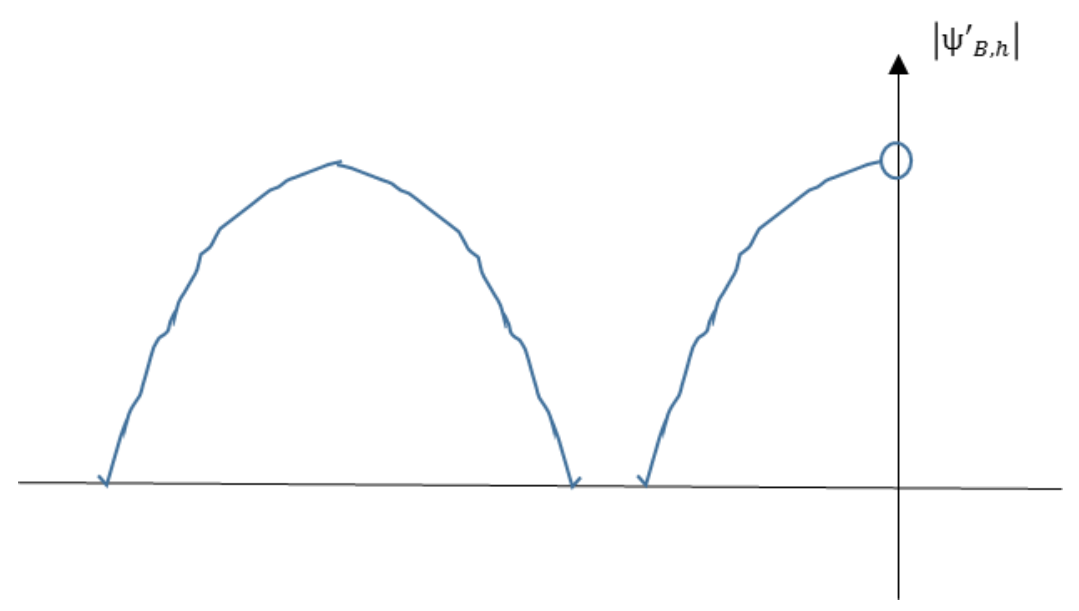

Fig. 10

Schematic of the entire wavefunction of the holes in the two-qubit case. Two constant phases appear. Note that the phases at the origin should be distinguished because the wavefunctions of the electrons and holes are discontinuous there. Thus there are right- and left-limits near the origin.

\subsubsection{NAND gate}

Fig. 11 illustrates a NAND gate using the proposed quantum diode system. In this figure, $\left(a_{i}, a_{j}\right)$ and $\left(b_{i}, b_{j}\right)$ are the input taps, and $\left(p_{i}, p_{j}\right)$ are the output taps. For example, when taps $\left(a_{i}, a_{j}\right)$ receive a voltage, they are turned ON, whereas taps $\left(a_{i}, a_{j}\right)$ receive a shorted current density are turned OFF. Note that the directions of the voltage and current density are not considered here. Moreover, another dual NAND gate exists in the B system. The important considerations are as follows:

1) An electrostatic potential from a voltage source is input between the corresponding taps of the $\mathrm{A}$ and B systems.

2) A shorted current appears between the corresponding taps of the A and B systems.

3) When both taps $\left(a_{i}, a_{j}\right)$ and $\left(b_{i}, b_{j}\right)$ are $\mathrm{ON}$, taps $\left(p_{i}, p_{j}\right)$ in the A system are shorted to the corresponding taps of another NAND gate in the B system.

The Results section tabulates the truth values of the output $\left(p_{i}, p_{j}\right)$, assuming that the bias current $j_{t 0}$ is ON. 


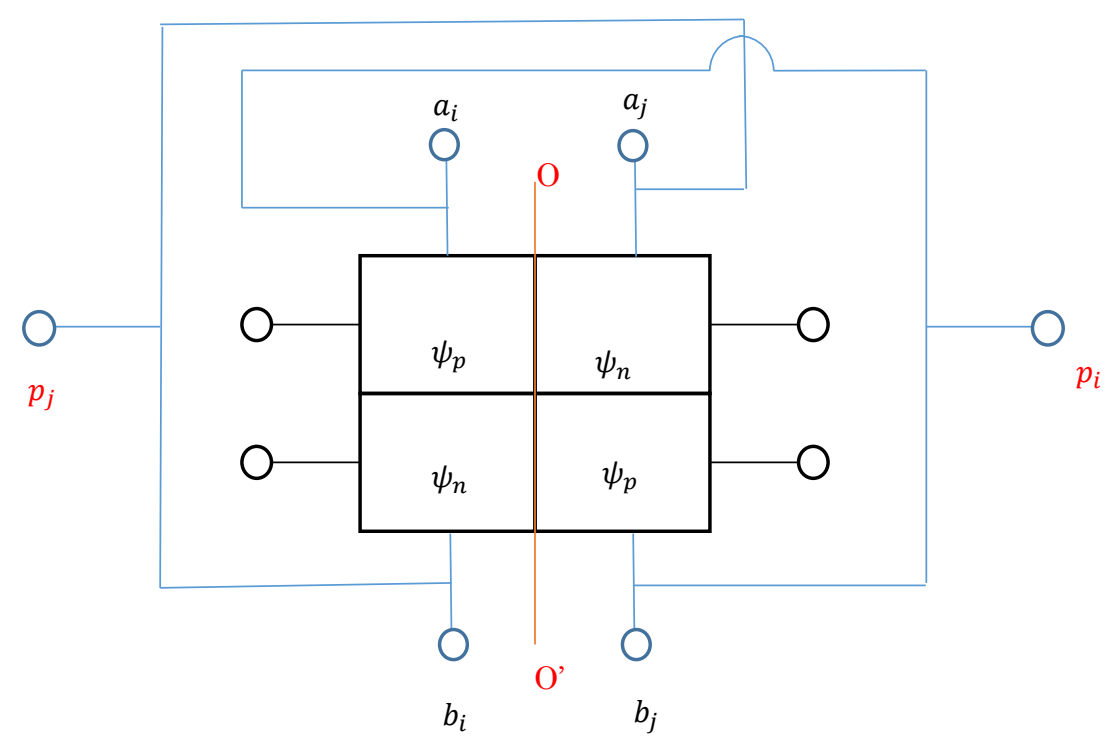

A system

Fig. 11 Schematic of a NAND gate. $\left(a_{i}, a_{j}\right)$ and $\left(b_{i}, b_{j}\right)$ are the input taps and $\left(p_{i}, p_{j}\right)$ are the output taps. This NAND gate resides in the A system (a dual NAND gate resides in the B system).

\subsection{Bias current density and initialization of information}

Let us revisit the NOT gate in the A system in the one-qubit case. First, when the bias current density $j_{t \mathrm{o}}$ is on and $\left(b_{i}, b_{j}\right)$ is also ON (i.e., receiving a voltage), then $\left(a_{i}, a_{j}\right)$ is turned ON. Tap $\left(a_{i}, a_{j}\right)$ can be turned OFF by a shorted current from the A system to the B system. This current density is counteracted by a dual reverse current from the B system to the A system passing an input voltage source. When the bias current density $j_{t 0}$ is off and a voltage is input to $\left(b_{i}, b_{j}\right)$, then $\left(a_{i}, a_{j}\right)$ exhibits no reverse voltage, because the junction $\mathrm{O}-\mathrm{O}$ ' fails the superconductivity condition.

When the voltages of $\left(b_{i}, b_{j}\right)$ and $\left(a_{i}, a_{j}\right)$ are ON, suppose that the bias current density $j_{t 0}$ is turned OFF in both the A and B systems. In this case, although $\left(a_{i}, a_{j}\right)$ is shorted, a persistent and steady output current density does not exist. Instead, the current decays because the superconductivity disappears, so the information of $\left(a_{i}, a_{j}\right)$ is eventually lost. Note that to obtain a steady output current density, the electric potentials' inputs from the voltage sources must be provided; otherwise, the output taps receive no power in view of energy conservation and the output current density must decay. This implies that the information of $\left(a_{i}, a_{j}\right)$ is initialized. 


\section{Results}

Note that, in simulations of this paper, a MS excel software was employed.

\subsection{Switching properties of the quantum diodes}

Figs. 12 and 13 display the current-density states from off to steady and from steady to off, respectively. In both instances, the switching speed is of the order of $10^{-14} \mathrm{~s}$, much faster than in conventional $\mathrm{p}-\mathrm{n}$ diodes.

Note that to apply the equation derived in the theoretical section, we must estimate the coherence $\xi$ of a Cooper pair.

Considering the energy gap discussed previously, we have

$$
\mathrm{e} \varphi_{B} \alpha=\hbar \omega
$$

which includes the fine structure constant $\alpha$. Details are given in the Discussion section.

Assuming the derived wavelength as the coherence of a Cooper pair, we obtain

$$
\lambda \equiv \xi=\frac{\hbar c}{e \varphi_{B}} \frac{1}{\alpha} \approx 7.3 \times 10^{-6} \mathrm{~m},
$$

where the bias potential $\varphi_{B} \equiv 3.7 \mathrm{~V}$.

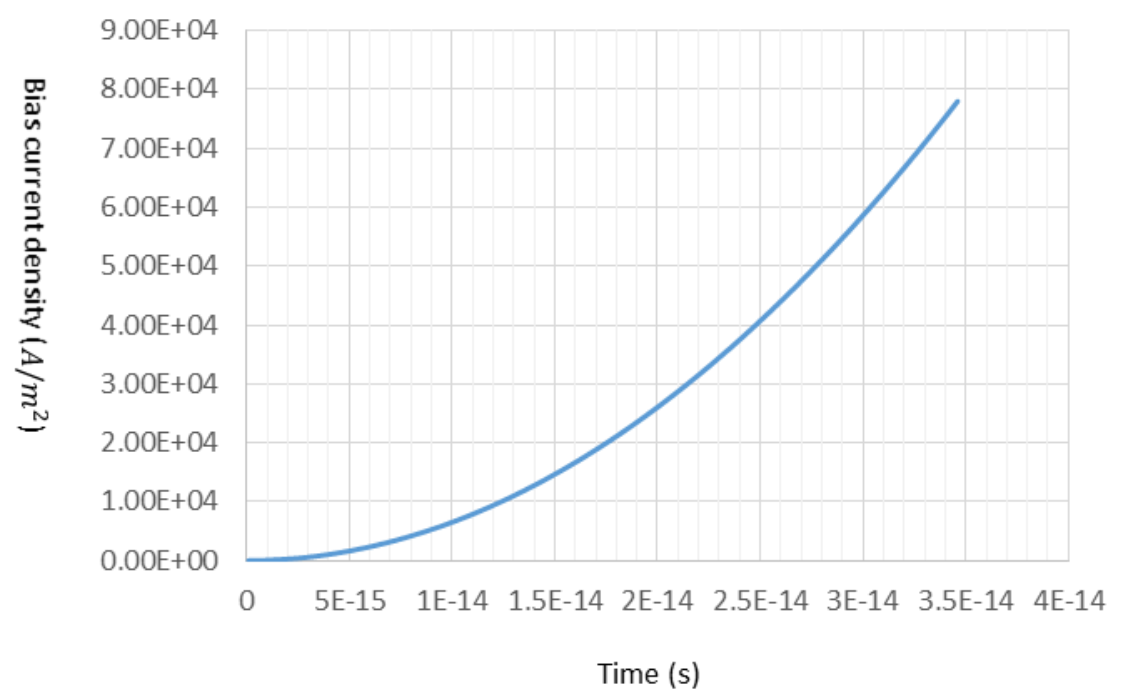

Fig. 12 Bias current density $j_{t}$ versus time (from off to steady state). The quantum diode switches in approximately $3.5 \times 10^{-14} \mathrm{~s}$. 


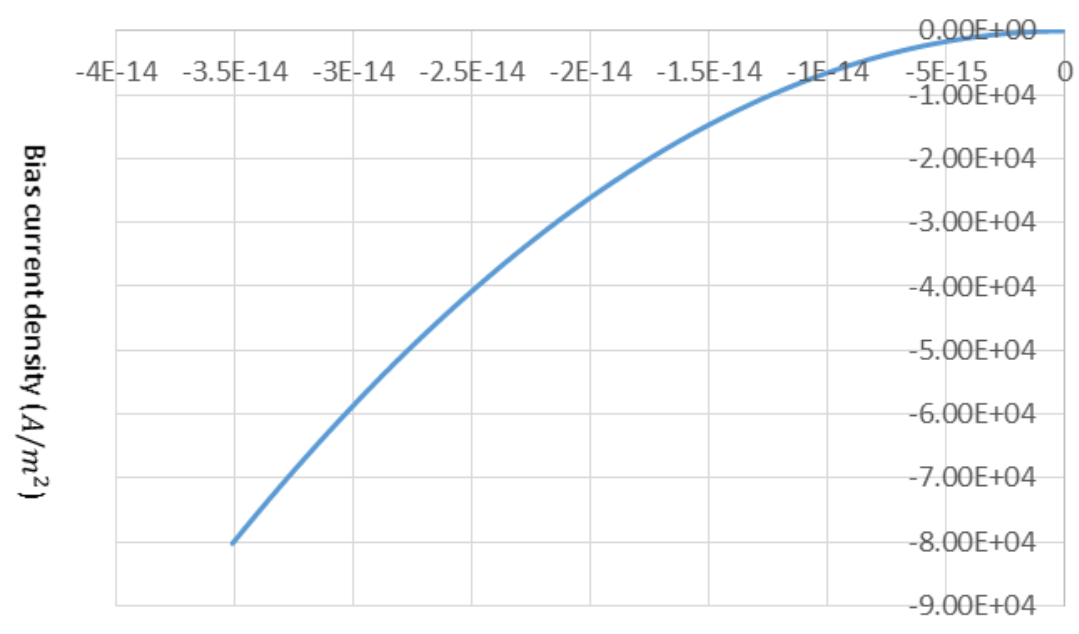

Time (s)

Fig. 13 Bias current density vs. time (from steady state to off). The switching time is that in Fig. 12.

\subsection{Many-body wavefunctions in the one-qubit case}

Figs. 15 and 16 show the wavefunctions of the electrons and holes, respectively determined by Eqs. (19) and (20), respectively. In both plots, the phases converge to constant values. Note that $\left|\psi_{h}\right|$ and $\left|\psi_{e}\right|$ contained in Eqs. (19) and (20) were given same and small fitting values in order to reproduce normalization of the total wavefuntion by Eq. (15) and (16).

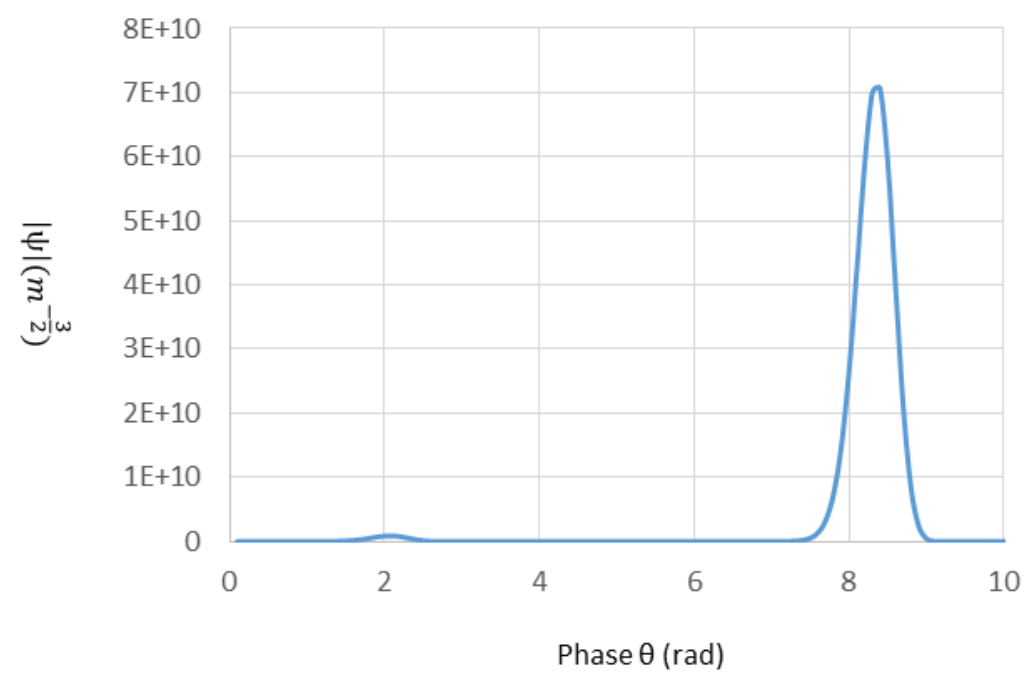

Fig. 15 Entire wavefunction of electrons. The wavefunction converges at 8.4 rad. 


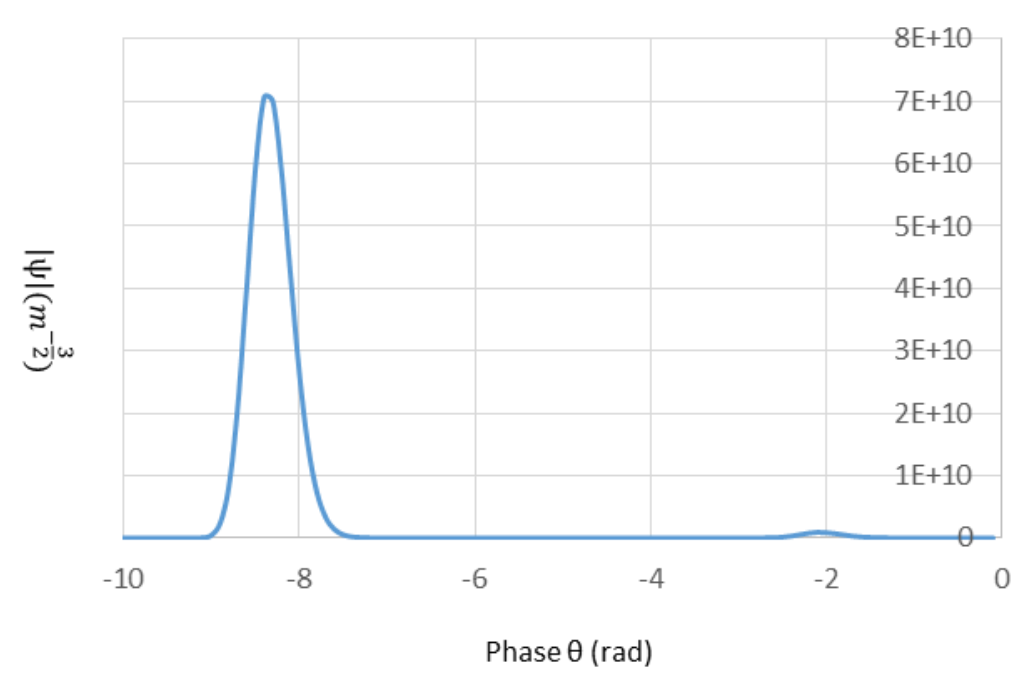

Fig. 16 Entire wavefunction for holes. The wavefunction converges at $\mathbf{- 8 . 4} \mathbf{r a d}$.

\subsection{Many-body wavefunctions in the two-qubit case}

Figs. 17 and 18 show the entire wavefunction of the electrons and holes, respectively, in the two-qubit case. Both plots show two converged quantum states. In Eq. (50), the value of $\left|\psi_{B, e}\right|$ and $\left|\psi_{B, h}\right|$ were determined by Eq. (15) and (16).

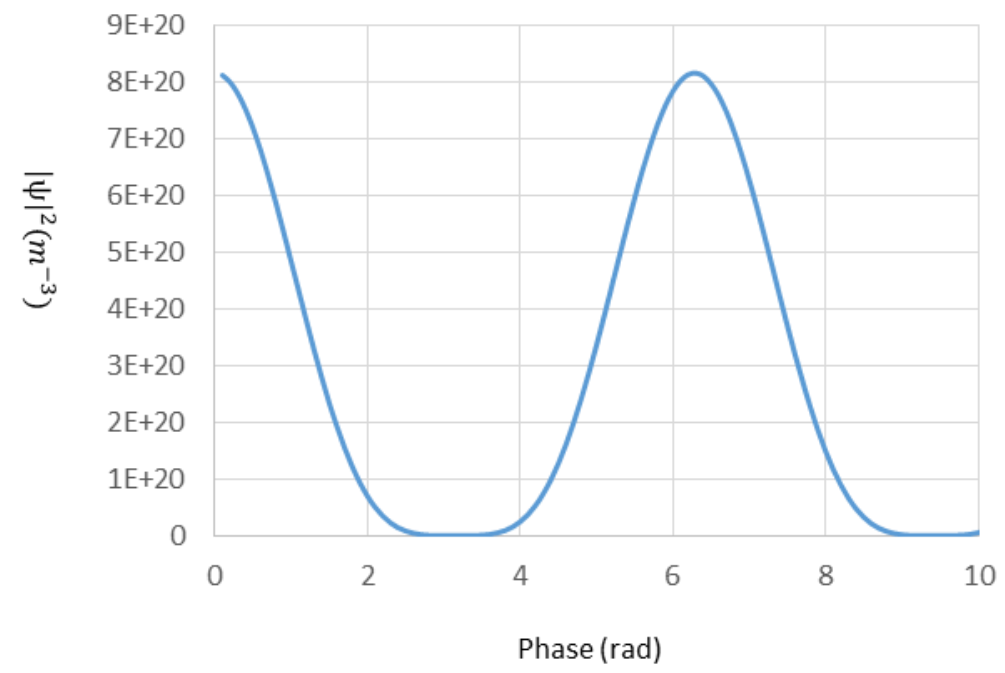

Fig.17 Entire wavefunction of the electrons in the two-qubit case. The entire wavefunction converges to two phases. 


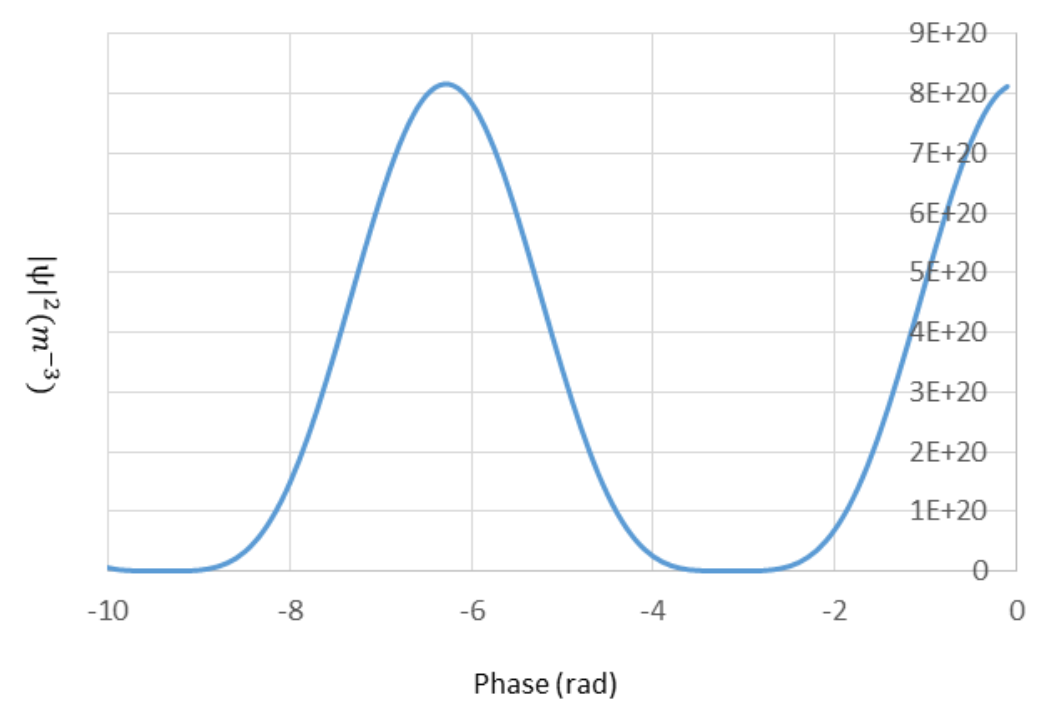

Fig. 18 Entire wavefunction of the holes in the two-qubit case. The sign of the converged phase opposite that in Fig. 17. Note that Figs. 17 and 18 are discontinuous at the origin because they are plotted for different carries. That is, near the origin, there are right- and left-limits.

\subsection{Result of NAND gate}

Table 1 shows the truth values of the derived NAND gate.

Table 1 Truth values of the derived NAND gate

\begin{tabular}{|c|c|c|}
\hline$\left(\begin{array}{ll}p_{i} & p_{j}\end{array}\right)$ & $\left(\begin{array}{ll}b_{i} & b_{j}\end{array}\right)$ & $\left(\begin{array}{ll}a_{i} & a_{j}\end{array}\right)$ \\
\hline off & on & on \\
\hline on & off & off \\
\hline on & off & on \\
\hline on & on & off \\
\hline
\end{tabular}

\section{Discussion}

5.1 Bias current, its superconductivity and the Meissner effect

To discuss general superconductivity, we must clarify the force that combines two carries into a Cooper pair. As mentioned, the combining force between an electron and a hole is assumed to be the attractive 
Coulomb force. As reported in our previous papers [21-22], the electromagnetic potential related to this attractive Coulomb force is not strictly equivalent to an energy gap that corresponds to energy of a photon, which has a momentum. To convert this potential to a photon energy, we introduce the fine structure constant $\alpha$ to the potential. Thus the required voltage (i.e., energy gap) is

$$
V=\mathrm{e} \varphi_{B} \times \alpha
$$

The critical bias potential $\varphi_{B, \text { crital }}$ at room temperature is obtained as

$$
\mathrm{e} \varphi_{B, \text { critical }} \times \alpha \approx k_{B} T,
$$

where $k_{B}$ denotes the Boltzmann constant, and the temperature $T$ is $300 \mathrm{~K}$.

Performing this calculation, we get

$$
\varphi_{B, \text { critical }} \approx 3.7 \mathrm{~V} \text {. }
$$

The applied electric potential must exceed $\varphi_{B, \text { critical }}$.

Let us discuss the deeper mechanism of superconductivity in terms of the bias current. Most specialists consider that superconductivity is automatically implied by a macroscopic and entire wavefunction with constant phase, i.e., the Meissner effect. In the present paper, however, we attempt to obtain a London equation, which provides direct evidence of the Meissner effect.

The numerical calculation revealed that the many-body wavefunctions, Eqs. (19) and (20) converged to macroscopic plane waves with a constant phase $\pm \theta_{0}$. Under an applied magnetic field $\boldsymbol{B}$ (i.e., vector potential $\boldsymbol{A}$ ), we can derive the Aharonov-Bohm (AB) effect [23] from the initial macroscopic wavefunction of electrons

$$
\psi_{B, e} \rightarrow \psi_{A}=|\psi| \exp \left[\left(\theta_{0}+\frac{2 q}{\hbar} \int A d s\right) j\right\},
$$

where $q, j$ and $\theta_{0}$ denote the electron charge, imaginary unit and converged phase of the macroscopic wavefunction, respectively.

Note that this section considers only the one-qubit system.

From Eq. (62), we have

$$
\left(\theta_{0}+\frac{2 q}{\hbar} \int A d s\right)=2 n \pi,
$$

where $n$ is an integer. Putting $n=0$, we have

$$
\theta_{0}=-\frac{2 q}{\hbar} \int A d s,
$$

and considering the center-of -mass motion of a pair,

$$
\theta_{0}=2 k_{0} x
$$

where $x$ is a variable.

Substituting Eq. (65) in Eq. (64) and differentiating both sides of Eq. (64), we obtain

$$
k_{0}=-\frac{q}{\hbar} A \text {. }
$$

The probability density flow is given by

$$
j_{S}=q|\psi|^{2} \frac{\hbar k_{0}}{m} .
$$


Substituting Eq. (66) in Eq. (67), we derive the following London equation:

$j_{s}=-q^{2}|\psi|^{2} \frac{A}{m}$.

This is a result from GL equation [24].

\subsection{Switching speed and memorization}

As indicated in the Results section, the switching speed of the designed quantum diode was of the order of $10^{-14} \mathrm{~s}$, substantially faster than that of conventional semiconductor devices (with ns-order switching times). Although this system has quantum supremacy and quantum correlation, it generally requires data initialization by switching the bias current $j_{t 0}$. Initialization is needed when mounting the quantum diodes and systems in an actual computer. Although quantum computing is usually limited by the clone prohibition theorem, memory in the proposed system is retained by the bias current density. Therefore, the quantum diode is compatible with conventional and existing programming, and quantum algorithms are not necessarily needed. Furthermore, owing to the much faster switching speed of the quantum diode than of conventional diodes, a computer constructed from such quantum diodes would operate much faster than conventional computers with the same programming methods.

5.3 Energy consumptions of the output current density and bias superconducting current density in the NOT gate

The currents in the system are the bias current and output currents. As mentioned above, the bias current is a superconducting current that generates no Joule heating or other energy losses. Furthermore, because the divergent output currents from the A system to the B system are shorted and unassociated with a voltage, they also generate no Joule heating. However, a simultaneous dual divergent current density appears, which reversibly passes the input voltage source from the B system to the A system. Because this current density is associated with the voltage from the voltage source, electric power is consumed. No other energy is consumed in the proposed system. The input potentials must be less than the bias electric potential (with a critical value of $\varphi_{B, \text { critical }} \approx 3.7 \mathrm{~V}$ ), but the magnitude of the divergent current was not estimated. Allowing an approximate estimation and assuming that the output divergent current is proportional to the input electric potential, a critical bias of $3.7 \mathrm{~V}$ permits an input electric potential of the order of $100 \mathrm{mV}$. Therefore, the corresponding divergent output current density will be of the order of $10^{3} \mathrm{~A} / \mathrm{m}^{2}$.

\subsection{Quantum supremacy and harvesting data}

In the two-qubit system, all electric potentials at all taps (i.e., all classical bits) can be input 
simultaneously and quantum correlations can form in the system. This paper claims that direct quantum correlations are possible, thus avoiding the extra overhead of indirect correlations. Furthermore, although a tap outputs a classical bit (a divergent current), the dual divergent input current passing the voltage source maintains the state of the quantum correlation, provided that the superconductivity, bias, and memory are maintained.

\subsection{Complete design of gate elements using NOT and NAND gates}

This paper proposed quantum NOT and NAND gates. Because these gates are very stable against relatively large bias voltages, they should be configurable into other gates such as AND gates. Therefore, the developed quantum diodes are expected to realize large-scale quantum computers with quantum correlations and quantum supremacy, which might completely replace existing computers.

\subsection{Bias and input electric potentials}

As mentioned, the input electric potential should not exceed the electric potential of the bias. If multiple input electric potentials dominate over the bias potential, the superconductivity is broken. Analogously, conventional and existing electric circuits require a bias DC voltage to convey a lowermagnitude AC signal.

\subsection{Compact size and required carrier number for superconductivity}

Reducing the gate size is a main focus of computing and semiconductor research. The ideal dimensions will contain a countable number of carriers. However, superconductivity requires a certain number of carriers are needed. In our numerical calculations, approximately 100 carriers converged the manybody wavefunctions (Eqs. (19) or (20)) to a constant phase. This carrier number is within the mesoscopic realm, implying that the quantum diode system can realize compact size in the mesoscopic realm.

\subsection{EPR pairs}

As mentioned, our quantum diodes are functional only when the A and B systems cooperate as dual systems. When a small voltage (small electric potential) is input between the A and B systems and the output state of the A system is determined, an output state of the B system is simultaneously and immediately determined as the dual state. We identify these dual states as an Einstein-PodolskyRosen pair [25-26]. 


\subsection{Stability to random and thermal noises}

The bias must arbitrarily exceed $3.7 \mathrm{~V}$, so an input electric potential smaller than the bias can itself be very large. As mentioned above, the critical bias allows a $100 \mathrm{mV}$-order input and an output of order $10^{3} \mathrm{~A} / \mathrm{m}^{2}$. If the bias is stable and sufficiently large, noises can be ignored, as their magnitudes will be much smaller than the input and output signals. Thus this quantum diode should operate stably even when mounted in a relatively large-scale computer.

\subsection{Significances of the presented contents}

The most significant contributions of this paper are summarized below

1) A new room-temperature superconductivity has been introduced to condensed matter physics.

2) A superconducting system with potential applicability to quantum computing has been proposed. Very recent quantum computing machines developed by other researchers are based on the existing refrigerated superconductors. Although these machines have technical merits (i.e. near-zero Joule heating losses, requiring only the refrigerating and running costs), the refrigerating costs are not low. Moreover, the liquid helium used for cooling metal superconductors is a limited resource on Earth. In contrast, the proposed system requires no refrigeration and is downsizable to small gate-elements. Because it accommodates many elements in a compact size and incurs low running costs, the system promises a departure from the existing computer types.

3) The proposed quantum diodes can be produced from the cheapest $\mathrm{p}-\mathrm{n}$ silicon based diodes, it can be fabricated by the manufacturing technologies of conventional diodes, which have accumulated since the onset of $\mathrm{p}-\mathrm{n}$ diodes and transistors. Therefore, the manufacturing cost of a computer constructed from the proposed quantum diodes should be very low. Considering that the superconducting bias current is generated without cooling, the low manufacturing costs should be matched by low running costs.

\section{Conclusions}

This paper presented a new superconductivity phenomenon that requires no refrigeration, and developed novel quantum diode systems. The new superconductivity was verified in numerical and analytical calculations. NOT and NAND gates, composed with many qubits and quantum correlations, promised to overcome almost all of the problems faced by current quantum computing research. The developments are expected to be shortly implemented in practice, as they incur low manufacturing and running costs. 
As a natural follow-up, the presented theory should be investigated in further experiments.

\section{Additional information}

This paper is unrelated to any competing interests such as funding, employment and personal financial interests. Moreover, it is unrelated to any non-financial competing interests.

\section{Acknowledgement}

1. We thank Enago (www.enago.jp) for English language Review.

2. I appreciate that this paper has been published as a preprint of MDPI as the follows: https://www.preprints.org/manuscript/202008.0577/v1

\section{References}

[1] B. Walsh. TIME, April 14 (2013)

[2] F. A .Zwanenbrug, et al, Rev. Mod. Phys. 85, 961(2013)

[3] J. P. Gaebler, et al, Phys. Rev. Lett., 117, 060505 (2016)

[4]C. J. Balance, et al, Phys. Rev. Lett., 117, 060504 (2016)

[5] R. Barends, et al, Nature, 508, 500-503 (2014)

[6] S. Sheldon, et al, Phys. Rev. A, 93, 060302 (2016)

[7] V. S. Denchev, et al, Phys. Rev. Lett. 117, 180402 (2016)

[8] T. Albash and D. A. Lidar, arXiv: 1705.07452

[9] M. Friesen, et al, Phys. Rev. Lett. 98, 230503 (2007)

[10] N. Y. Yao, et al, Phys. Rev. Lett. 106, 040505 (2011)

[11] M. Yamaguchi, et al., IEEE Transactions on Applied Superconductivity. 13 (2), 1848-1851 (2003)

[12] J. Bardeen, L.N. Cooper and J. R. Schrieffer, Phys. Rev. 108,(5), 1175-1204 (1957)

[13] J.G. Bednorz and K.A. Müller, Zeitschrift für Physik B. 64, 189-193 (1986)

[14] Y. Kamihara, et al, J. Am. Chem. Soc, 128 (31), 10012-10013 (2006)

[15] J. Nagamatsu, et al, Nature, 410, 63 (2001)

[16] M. Somayazulu, et al, Phys. Rev. Lett. 122, 027001 (2019)

[17] S. Ishiguri, · Journal of Superconductivity and Novel Magnetism 24 (1), 455-462 (2011)

[18] S. Ishiguri,, "Theory on Another Type of Temperature-Independent Superconductivity Based on Circuit Approaches with High Critical Current Density" Preprints 2019, 2019110033 (doi: 10.20944/preprints201911.0033.v2). 
[19] S. Ishiguri, J Multidis Res Rev, 2(1), 69-80 (2020)

[20] S. Ishiguri, "New Superconductivity and Theoretical Study on a New Phenomenon of Energy Source with Assistance of Initial Experiments", Preprints 2018, 2018110636 (doi: 10.20944/preprints201811.0636.v1).

[21] S. Ishiguri, "Analytical Descriptions of High-Tc Cuprates by Introducing Rotating Holes and a New Model to Handle Many-Body Interactions", Preprints 2020, 2020050105 (doi: 10.20944/preprints202005.0105.v1).

[22] S. Ishiguri, "Unified Field Theory for Electromagnetic and Gravity Fields with the Introduction of Quantized Space-Time and Zero-Point Energy”, Preprints 2020, 2020070462.

[23] Y. Aharonov and D. Bohm, Phys. Rev. 115, 485 (1959)

[24] C. Kittel, Introduction to Solid State Physics (Japanese 8th-edition), Appendix I, Maruzen in Tokyo, (2010)

[25] C. H. Bennett, et al, Phys .Rev. Lett. 70(13), 1895-1899 (1993)

[26] A. Einstein, et al, Phys. Rev. 47, 777 (1935) 